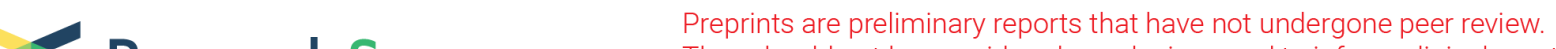 $\begin{array}{ll}\text { Research Square } & \begin{array}{l}\text { They should not be considered conclusive, used to inform clinical practice, } \\ \text { or referenced by the media as validated information. }\end{array}\end{array}$
}

\section{Elsberg Syndrome in the Setting of Asymptomatic SARS-CoV-2 infection: Case Report}

\section{Rory M. C. Abrams ( $\sim$ Rory.Abrams@mssm.edu )}

Icahn School of Medicine at Mount Sinai https://orcid.org/0000-0002-9675-4012

Fiona Desland

Icahn School of Medicine at Mount Sinai

Helaina Lehrer

Icahn School of Medicine at Mount Sinai

Anne Yeung

Icahn School of Medicine at Mount Sinai

Winona Tse

Icahn School of Medicine at Mount Sinai

James J. Young

Icahn School of Medicine at Mount Sinai

Damodara R. Mendu

Icahn School of Medicine at Mount Sinai

Barbara G. Vickrey

Icahn School of Medicine at Mount Sinai

Susan C. Shin

Icahn School of Medicine at Mount Sinai

\section{Case Report}

Keywords: Coronavirus-19, SARS-CoV-2, neuromuscular disorders, Elsberg syndrome, polyneuropathy

Posted Date: October 30th, 2020

DOI: https://doi.org/10.21203/rs.3.rs-98567/v1

License: (c) (i) This work is licensed under a Creative Commons Attribution 4.0 International License. Read Full License

Version of Record: A version of this preprint was published at Journal of Clinical Neuromuscular Disease on October 30th, 2020. See the published version at https://doi.org/10.1097/CND.0000000000000369. 


\section{Abstract}

Background: Elsberg syndrome is a rare cause of lumbosacral radiculitis with concomitant thoracic or lumbosacral myelitis that can be seen following an acute or reactivated viral infection. COVID-19 disease, caused by the SARS-CoV-2 virus, has quickly spread to a global pandemic since its first discovery in the winter of 2019. During this time, there has been an increasing number of case reports describing SARSCoV-2 associated neuroinflammatory disease.

Case Presentation: A 68-year-old man presented in June 2020 with a fall due to progressive lower extremity weakness and numbness, occurring shortly after the initial coronavirus surge in New York City. He developed ascending numbness to the level of the lower abdomen over the preceding month. He subsequently experienced low back pain, and gastrointestinal and genitourinary dysfunction. An extensive laboratory and radiologic evaluation ensued which was notable for elevated SARS-CoV-2 IgG antibodies despite an absence of preceding COVID-19 symptoms. Initial electrodiagnostic testing was notable for absent late responses in the lower extremity nerve conductions with normal distal sensorimotor conductions, and incomplete muscle activation with otherwise normal motor unit morphology and recruitment on electromyography. Repeat testing two weeks later revealed similar nerve conductions, but also the interval development of active neurogenic changes and reduced motor unit recruitment in the L3-L4 myotomes. This was suggestive of a lower thoracic and lumbosacral myelopathy and lumbosacral polyradiculopathy without peripheral neuropathy. A diagnosis of Elsberg syndrome was made and treatment with intravenous methylprednisolone yielded mild clinical improvement and the electrodiagnostic re-emergence of the lower extremity late responses.

Conclusions: We report here, to our knowledge, the first case of suspected COVID-19 associated Elsberg syndrome, which may help to shed light on ways in which to approach diagnostic and treatment options in COVID-19 patients presenting with uncommon neurological and autonomic manifestations.

\section{Background}

Elsberg syndrome is a rare cause of lumbosacral radiculitis with concomitant thoracic or lumbosacral myelitis that presents following an acute or reactivated viral infection. This syndrome is most commonly associated with herpes simplex virus 2 (HSV2) infection $(1,2)$ but has also been reported following acute West Nile virus (WNV) infection (3-6). COVID-19 disease caused by the SARS-CoV-2 virus was first described in December 2019 in Wuhan, China, but has since rapidly spread globally. An increasing number of case reports have described SARS-CoV-2 infection that may yield associated myelitis, GuillainBarré syndrome (GBS), meningitis, and CNS vasculitis (7-9). It has been suggested that the severe systemic sequelae of COVID-19 disease might be due to aberrant interferon responses, leading to an excessive hyperinflammatory response (10). Therefore, unlike in HSV2 and WNV associated disease, much of the tissue damage associated with SARS-CoV-2 infection is thought to be mediated by an excessive post-viral inflammatory host response $(10,11)$. Herein, we report a suspected case of Elsberg 
syndrome, representing a unique neuromuscular entity within a spectrum neuroinflammatory disease that may be attributable to a post-COVID-19 state.

\section{Case Presentation}

A 68-year-old man presented in June 2020 to the emergency room with a fall due to progressive lower extremity weakness and numbness, occurring shortly after the peak of the initial coronavirus surge in New York City. The patient had a history of benign prostatic hyperplasia and hyperlipidemia. He reported that beginning one month prior to presentation he developed diminished sensation in his feet. Over the course of several weeks the numbness progressively ascended to the level of the lower abdomen and included the perianal region. Subsequently, he developed low back pain, urinary retention, and constipation. He reported experiencing several falls due to his weakness and sensory loss, which culminated in a mechanical fall whereby he sustained a left distal fibular fracture, prompting medical attention. He denied any visual changes, headaches, dizziness, shortness of breath, fatigue, arthralgias, myalgias, fevers, loss of smell or taste, or any recent upper respiratory or gastrointestinal illness; he never had symptoms suggestive of COVID-19 disease and was never previously tested for it. He denied recent travel with his last trip being to Europe and Israel in the 1980s.

Upon admission, the patient underwent casting of the fibular fracture and a foley catheter was placed for his urinary retention. His physical examination was notable for abdominal distension with moderate tenderness and stool burden in the left lower quadrant. Neurologic examination showed he was fully oriented with fluent, clear speech and normal cranial nerve exam. He had normal bulk, tone, and confrontation strength in both upper extremities. There was right lower extremity diminished tone, and assessment of the left lower extremity was limited due to the casting. There was Medical Research Council (MRC) grade 2/5 hip flexion strength bilaterally, 4+/5 knee extension bilaterally, 2/5 knee flexion bilaterally, and $4 / 5$ ankle dorsiflexion and plantar flexion on the right. Deep tendon reflexes were notable for $3+$ right patellar responses, bilateral crossed adductors, absent right Achilles reflex, and upgoing plantar responses on the right. Multimodal sensation testing was normal in the upper extremities, however, there was dense sensory loss to all modalities including proprioception below the approximate T8 spinal level. Given a reproducible spinal sensory level, initial considerations focused on a myelopathic process.

Work up in the emergency department included MRIs of the cervical, thoracic, and lumbar spine which showed areas of cervical and thoracic spondylosis and mild cervical and lumbar canal narrowing without cord signal changes. These chronic findings were felt unlikely to explain the patient's acute symptoms. He was admitted to the neurology service for further evaluation and management. The patient underwent extensive additional neuraxial imaging including repeat contrast MRIs of the spine that showed a potential subtle hyperintense signal in the dorsal cord at T10 (Fig 1A). Given the persistent concern for a 
myelopathic process, a spinal MR angiogram and two conventional spinal angiograms were performed which failed to demonstrate vascular abnormalities of the spinal cord and dura. An MRI brain incidentally showed non-specific faint bilateral, symmetric T2/FLAIR hyperintensities in the brainstem surrounding the fourth ventricle. These findings were felt likely metabolic in etiology, although Wallerian degeneration of the upper motor neuron was also considered.

The serum laboratory workup was notable for elevated SARS-CoV-2 IgG antibody (12) (titer of 1:2880), elevated C-reactive protein (31.6 mg/L) and erythrocyte sedimentation rate $(22 \mathrm{~mm} / \mathrm{hr})$. Nasopharyngeal swab for SARS-CoV-2 PCR was negative. Serum angiotensin converting enzyme, complement C3/C4, creatinine kinase, TSH, Vitamin B12, Vitamin E, copper, zinc, thiamine, fatty acids, RPR, EBV PCR, and enterovirus RNA PCR were not detected or within normal limits. Other antibody studies including antiMOG and NMO IgG, anti-SSA/SSB, ANA, ANCA, anti-DNA, anti-Hu, rheumatoid factor, anti-mycoplasma, Lyme IgG/lgM, hepatitis A/B IgG/IgM, ant-hepatitis C, HIV IgG/lgM, HTLV I/II, Polio types 1 and 3, VZV $\lg$, West Nile Virus IgG/IgM, autoimmune encephalopathy and motor sensory neuropathy/ganglioside panels were negative.

Lumbar puncture was performed three weeks after his fall. CSF profile revealed 10 red blood cells $/ \mu \mathrm{L}, 0$ white blood cells $/ \mu \mathrm{L}$, glucose $62 \mathrm{mg} / \mathrm{dL}$, and protein at $38 \mathrm{mg} / \mathrm{dL}$. An incidental positive West Nile Virus IgG antibody was detected, but CSF was negative for West Nile Virus IgM antibodies and PCR. Additional infectious and inflammatory workup was also negative for bacterial, fungal, and viral cultures, Biofire PCR panel, enterovirus PCRs, oligoclonal bands, and autoimmune encephalopathy antibody panel. SARSCoV-2 viral PCR and antibodies were also not detected in the CSF.

The patient underwent serial nerve conduction studies (NCS) and electromyography (EMG). The initial NCS were performed three weeks following his mechanical fall. This study revealed normal distal motor and sensory conductions in the upper and right lower extremities without evidence for conduction block or slowing; however, the tibial and peroneal F-wave responses and the tibial H-reflex responses were absent in the right lower extremity. EMG revealed incomplete muscle activation, but normal recruitment pattern and motor unit morphology. Repeat electrodiagnostic studies two weeks later again showed normal distal motor and sensory conductions. The repeat EMG revealed the interval development of spontaneous activity in the right vastus lateralis, iliacus, and paraspinal muscles. A single volitional motor unit of normal amplitude and duration was seen rapidly firing within the iliacus muscle, and other muscles were limited by activation. 
The initial EMG results suggested a neurogenic process affecting the proximal nerve roots and or distal conus with a superimposed upper motor neuron problem causing an incomplete activation pattern (13). An active neurogenic process affecting the L3-L4 myotomes was confirmed on the second EMG by the presence of spontaneous activity as well as markedly reduced motor unit recruitment. This new data taken together with the patient's clinical picture, and markedly elevated SARS-CoV-2 antibodies, raised the suspicion for a post-COViD-19 immune-mediated myeloradiculitis, or Elsberg syndrome.

To target a presumed neuroinflammatory autoimmune response, the patient was started on intravenous methylprednisolone (IVMP) 1,000 mg daily for 5-days. In the days following the steroid course, the patient reported some subjective improvement in both weakness and numbness. There was concurrent clinical re-emergence of the right Achilles tendon reflex. Consistent with this finding, focused NCS at that time revealed electrodiagnostic re-emergence of the peroneal and tibial F-wave (Fig 1B) and H-reflex late responses in the patient's right lower extremity. Following completion of course of IVMP, the patient received a course of intravenous immunoglobulin (IVlg; $0.4 \mathrm{mg} / \mathrm{kg} /$ day for 5 -days). His hospitalization was complicated by obstipation causing bowel edema requiring disimpaction and aggressive laxative management. He was subsequently discharged to acute inpatient rehab. At the time of follow-up threemonths after symptom onset he continued to demonstrate marked paraparesis, sensory dysfunction, and bowel/bladder function, not significantly changed beyond the improvement achieved following steroid treatment.

\section{Discussion And Conclusion}

This is among the earliest reported cases of post-COVID-19 disease associated with acute onset neuromuscular dysfunction (14-18). This patient presented with a lower extremity paraparesis and dense sensory loss, associated with low back pain, and neurogenic bowel and bladder dysfunction. The presence of a lower thoracic sensory level on exam, the lower extremity weakness, and the bowel/bladder dysfunction was suggestive of a diffuse process involving the thoracic, lumbar, and sacral spinal cord segments, respectively. A clinical diagnosis of Elsberg syndrome was made based on the clinical picture of lower thoracic myelitis, functional cauda equina syndrome, with electrodiagnostic evidence of polyradiculitis without peripheral neuropathy (1). The diagnosis of Elsberg syndrome was challenging given the lack of obvious radiologic or angiographic evidence for myelitis and the rarity of this diagnosis.

Recent reports of acute- and post-COVID-19 disease associated myelitis $(7,9,19)$ and GBS have been made $(15,16,18,20)$. Importantly, Elsberg syndrome represents a unique neuroinflammatory disorder that is a distinct acute neuromuscular entity, along the continuum of either isolated transverse myelitis or the distal symmetric polyneuritis seen in GBS. GBS was excluded based on the multiple electrodiagnostic studies and by CSF-based criteria, specifically the lack of albuminocytologic dissociation (21). The clinical distinction between these disparate entities may have diagnostic, management, and prognostic 
implications. The delayed development of active neurogenic changes along the L3-L4 myotomes was suggestive of an ongoing process nearly two months from the earliest symptom onset. Remarkably, following IVMP the repeat EMG demonstrated re-emergence of the previously absent F-wave (Fig 1B) and $\mathrm{H}$-reflex late responses in the right lower extremity, supportive of the subjective clinical improvement. Unlike GBS, however, this suggests that post-COVID-19 Elsberg syndrome is a steroid responsive process (21). Despite the empiric courses of IVMP and IVIG, the clinical improvement was incomplete.

Conceivably, convalescent plasma collected from donors who have recovered from SARS-CoV-2 infection could be used as a possible therapy, but this therapy was deferred given the possibility of exacerbating his condition, should the donor plasma contain parainfectious antibodies that may further attack the central or peripheral nerves (22). This raises the issues of the optimal treatment and timing of therapy.

In addition to the elevated SARS-CoV-2 IgG antibodies in the blood, there were positive WNV IgG antibodies in the CSF. It is unlikely that the patient had recent West Nile infection given the absence of recent exposure risk factors, the epidemiologic peak being in the fall months, and the lack of serum PCR or antibodies (5), although a remote exposure may be possible. One interesting hypothesis to consider includes the possibility that the systemic inflammatory response to SARS-CoV-2 may have led to the reproduction of the WNV antibodies from a remote exposure. This combined inflammatory response could have potentiated a biomimicry response that specifically targeted neuronal tissues. However, due to the patient's presentation during the peak of the initial surge of SARS-CoV-2 infections in New York City, we believe an acute or post-infectious viral etiology due to recent SARS-CoV-2 infection was the initiating pathological event. Consistent with this, there were also the faint encephalitic changes seen on brain MRI T2/FLAIR sequences that may conceivably be related to this post-viral syndrome; however, these changes could be secondary to long-tract changes to the upper motor neuron. Given the challenging nature of this case with an already extensive workup, it may be difficult to confirm an exact etiology and pathophysiologic mechanism.

This case highlights an additional neurologic complication suspected to be attributable to asymptomatic SARS-CoV-2 infection that was not previously recognized. Earlier diagnosis of Elsberg syndrome in these patients may prompt sooner treatment, quicker recovery, and avoidance of medical complications from immobility and autonomic dysfunction. Given the rising global incidence of SARS-CoV-2 infection, raising awareness of the various neurologic manifestations in the post-COVID-19 state may augment patient outcomes.

\section{Abbreviations}

CNS - Central nervous system 
COVID-19 - Coronavirus disease of 2019

CSF - Cerebrospinal fluid

EMG - Electromyography

HSV2 - Herpes simplex virus 2

IVIg - Intravenous immunoglobulin

IVMP - Intravenous methylprednisolone

GBS - Guillain-Barré syndrome

MRC - Medical Research Council

NCS - Nerve conduction studies

WNV - West Nile virus

\section{Declarations}

As our manuscript reports data on a patient with COVID-19, we are confirming that this case has not been reported in any other submission or included in any other form by me, our group, or anyone else.

Ethics approval and consent to participate - Not applicable.

Consent for Publication - Informed written consent for the publication of case details, radiologic images, and relevant laboratory data was obtained from the patient, including personal details in forms written and online, which the authors have access to. The patient was informed that information was as deidentified as possible and would be available online. Additional consent for publication was received based on departmental and institutional review board (IRB) approval.

Availability of data and materials - The main patient data generated or analysed during this study are included in this published article. The data collected and/or analysed during the current report are available from the corresponding author on reasonable request.

Competing interests - The authors declare that they have no competing interests.

Funding - There are no funding sources relevant to this case report to declare. 


\section{References}

1. Savoldi F, Kaufmann TJ, Flanagan EP, Toledano M, Weinshenker BG. Elsberg syndrome: A rarely recognized cause of cauda equina syndrome and lower thoracic myelitis. Neurol Neuroimmunol Neuroinflamm. 2017;4(4):e355.

2. Eberhardt O, Kuker W, Dichgans J, Weller M. HSV-2 sacral radiculitis (Elsberg syndrome). Neurology. 2004;63(4):758-9.

3. Hawkes MA, Toledano M, Kaufmann TJ, Rabinstein AA. West Nile Neuroinvasive Disease Presenting as Elsberg Syndrome. Neurologist. 2018;23(5):152-4.

4. Leis AA, Stokic DS. Neuromuscular manifestations of west nile virus infection. Front Neurol. 2012;3:37.

5. Winkelmann ER, Luo H, Wang T. West Nile Virus Infection in the Central Nervous System. F1000Res. 2016;5.

6. Chan V, Minalyan A, Ottman P, Raza A, Tewary A. Falling for a Diagnosis: West Nile Myelitis without Encephalitis. Cureus. 2019;11(10):e5838.

7. Ellul MA, Benjamin L, Singh B, Lant S, Michael BD, Easton A, et al. Neurological associations of COVID-19. Lancet Neurol. 2020.

8. Munz M, Wessendorf S, Koretsis G, Tewald F, Baegi R, Kramer S, et al. Acute transverse myelitis after COVID-19 pneumonia. J Neurol. 2020;267(8):2196-7.

9. Sotoca J, Rodriguez-Alvarez Y. COVID-19-associated acute necrotizing myelitis. Neurol Neuroimmunol Neuroinflamm. 2020;7(5).

10. Vabret N, Britton GJ, Gruber C, Hegde S, Kim J, Kuksin M, et al. Immunology of COVID-19: Current State of the Science. Immunity. 2020;52(6):910-41.

11. Tay MZ, Poh CM, Renia L, MacAry PA, Ng LFP. The trinity of COVID-19: immunity, inflammation and intervention. Nat Rev Immunol. 2020;20(6):363-74.

12. Amanat $F$, Stadlbauer $D$, Strohmeier $S$, Nguyen THO, Chromikova V, McMahon M, et al. A serological assay to detect SARS-CoV-2 seroconversion in humans. Nat Med. 2020;26(7):1033-6.

13. Syme JA, Jr., Kelly JJ, Jr. Absent F-waves early in a case of transverse myelitis. Muscle Nerve. 1994;17(4):462-5.

14. Padroni M, Mastrangelo V, Asioli GM, Pavolucci L, Abu-Rumeileh S, Piscaglia MG, et al. Guillain-Barre syndrome following COVID-19: new infection, old complication? J Neurol. 2020;267(7):1877-9.

15. Zhao H, Shen D, Zhou H, Liu J, Chen S. Guillain-Barre syndrome associated with SARS-CoV-2 infection: causality or coincidence? Lancet Neurol. 2020. 
16. Toscano G, Palmerini F, Ravaglia S, Ruiz L, Invernizzi P, Cuzzoni MG, et al. Guillain-Barre Syndrome Associated with SARS-CoV-2. The New England journal of medicine. 2020.

17. Sedaghat Z, Karimi N. Guillain Barre syndrome associated with COVID-19 infection: A case report. J Clin Neurosci. 2020.

18. Gutierrez-Ortiz C, Mendez A, Rodrigo-Rey S, San Pedro-Murillo E, Bermejo-Guerrero L, Gordo-Manas R, et al. Miller Fisher Syndrome and polyneuritis cranialis in COVID-19. Neurology. 2020.

19. Guidon AC, Amato AA. COVID-19 and neuromuscular disorders. Neurology. 2020.

20. Uncini A, Vallat JM, Jacobs BC. Guillain-Barre syndrome in SARS-CoV-2 infection: an instant systematic review of the first six months of pandemic. J Neurol Neurosurg Psychiatry. 2020.

21. Wijdicks EF, Klein CJ. Guillain-Barre Syndrome. Mayo Clin Proc. 2017;92(3):467-79.

22. Zhao Q, He Y. Challenges of Convalescent Plasma Therapy on COVID-19. J Clin Virol. 2020;127:104358.

\section{Figures}

A

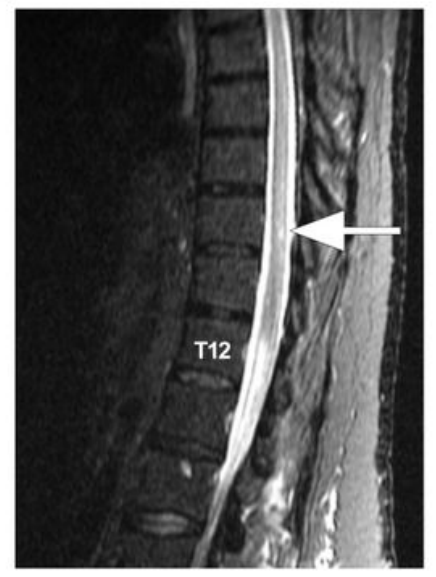

B

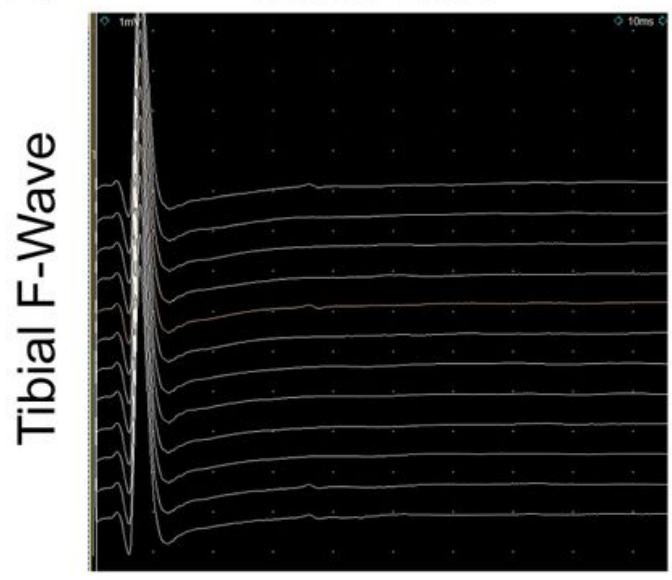

Follow-Up EMG

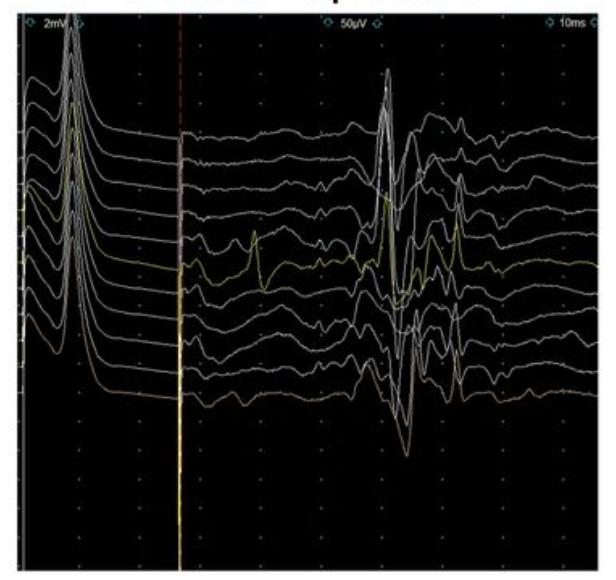

\section{Figure 1}

A. MRI T2/STIR-based sequences of the lumbosacral spine reveals focal intra-axial T2/FLAIR hyperintensity within the dorsal aspect of the spinal cord, maximal at approximately the T10 spinal level. The spinal cord is demonstrated to terminate at approximately T12. B. The right tibial F-wave responses recorded on the initial NCS (left) and repeat NCS following the steroid course (right). The lower extremity late responses were absent on the initial NCS performed three-weeks following his mechanical fall. The repeat NCS were performed on day 4/5 of IVMP in accordance with his subjective clinical improvement and return of his Achilles deep tendon reflexes. 